\title{
Rechazar y retirar el tratamiento al final de la vida: complejidades éticas que involucran a pacientes que carecen de capacidad para tomar decisiones
}

\section{Refusing and withdrawing treatment at the end of life: ethical complexities involving patients who lack decision-making capacity}

\begin{abstract}
Marie-Jo Thie/*
https://doi.org/10.36105/mye.2020v31n4.07

Resumen

Obtener el consentimiento válido, libre e informado no siempre es fácil. Presupone, por un lado, la divulgación de información justa, clara y apropiada, y, por otro, la capacidad de comprenderla lo más adecuadamente posible y luego tomar una decisión. Entonces, cuando un paciente tiene impedimentos cognitivos en el largo plazo y carece de la capacidad independiente para tomar o comunicar una decisión, y cuando esta decisión se trata del final de su vida, el consentimiento puede ser muy complejo. ¿Cómo hacerlo bien?

El presente artículo no ofrece «la» solución, pero analiza el tema en el marco legal y ético francés. El desafío es encontrar una línea divisoria entre la beneficencia, el respeto a la autonomía y el rechazo de la «obstinación irrazonable» (que define la
\end{abstract}

\footnotetext{
* Universidad de Strasbourg, Francia. Correo electrónico: mthiel@unistra.fr https://orcid.org/0002-9987-3383

Recepción: 20 de junio de 2020. Aceptación: 15 de julio de 2020.
} 
M-J. Thiel

inutilidad en la legislación francesa). La beneficencia podría significar retirar (o retener) los tratamientos médicos en curso (quimioterapia, radioterapia, etcétera) cuando se vuelven cada vez más ineficaces $y$, además, son agresivos e intrusivos para el paciente (lo que conlleva una disminución de su calidad de vida). Pero cuando el paciente no puede consentir o parece estar en desacuerdo, ¿cómo podemos seguir adelante?

La contribución examinará, primero, el valor de la toma de decisiones y del consentimiento del paciente en la atención médica y, luego, el papel que la familia y los cuidadores pueden desempeñar para apoyarlos. Dado que la muerte es un momento único y definitivo, no debemos olvidar que, a menudo, la experiencia de los familiares con el paciente condiciona tanto el proceso de toma de decisiones como su proceso de duelo. Finalmente, se examinarán y discutirán tres situaciones clínicas específicas, en las que se debe tomar una decisión con respecto a la retención y retirada de los tratamientos: cuando un paciente está consciente, cuando está inconsciente y no tiene instrucciones anticipadas, cuando rechaza el tratamiento a sabiendas. La lucha consiste en saber qué es lo que realmente le importa a la persona moribunda, para poder respetar sus deseos.

Palabras clave: consentimiento, instrucciones anticipadas, fideicomisarios, atención centrada en la familia, información médica.

Muy a menudo, las familias se enfrentan al final de la vida con la cuestión ética. No se trata de suspender la atención, que siempre se debe (1), sino de interrumpir los tratamientos médicos en curso, especialmente aquellos que son agresivos e intrusivos para el paciente. Dichos tratamientos (quimioterapia, radioterapia, cirugía, etcétera), pueden resultar en una disminución de la calidad de vida del paciente y volverse cada vez más ineficaces. La ley francesa (marco de este documento) describe esta situación de inutilidad con la expresión «obstinación irrazonable».

Retirar los tratamientos en este contexto puede ser éticamente controvertido cuando el paciente tiene deficiencias cognitivas de 
Rechazar y retirar el tratamiento al final de la vida: complejidades éticas...

largo plazo y carece de la capacidad independiente para tomar o comunicar una decisión (aproximadamente) al final de su vida. Si el paciente ha dado instrucciones anticipadas claras o ha designado a una persona de confianza para apoyarlo o representarlo, las decisiones con respecto a estos tratamientos podrían ser más fáciles. A veces, sin embargo, no hay instrucciones anticipadas. Los miembros de la familia podrían estar divididos al interpretar los deseos no declarados de su ser querido. El caso de Vincent Lambert, en Francia (2), es emblemático de esta situación. En otros casos, sin embargo, la familia del paciente ni siquiera está presente. ¿Cómo, entonces, resolvemos los problemas éticos relacionados con la retirada del tratamiento?

Las situaciones de fin de vida agravan las dificultades de obtener un consentimiento válido, libre e informado en el caso de personas con deficiencias cognitivas. Incluso sin tales impedimentos, no está claro cómo evitar todas las trampas del consentimiento sustituto y sus tergiversaciones, particularmente en el mundo anglosajón, donde los estudios que involucran a pacientes con capacidad de toma de decisiones y sus familiares señalan «en un tercio de los casos a un desajuste entre los deseos del paciente y los de su familia» (3). Esto resalta la importancia de tratar de saber lo que realmente le importa a la persona moribunda, y respetar sus deseos, valores, sentimientos y creencias con respecto a las decisiones de atención médica al final de la vida.

En este documento, primero examinaremos el valor de la toma de decisiones y el consentimiento del paciente en la atención médica y, luego, el papel que la familia y los cuidadores pueden desempeñar para apoyarlos. Propondré que la muerte es un momento único y definitivo, y que a menudo la experiencia de los familiares con el paciente condiciona tanto el proceso de toma de decisiones como su proceso de duelo. Finalmente, examinaré y analizaré tres situaciones clínicas específicas, en las que se debe tomar una decisión con respecto a la retención y la retirada de los tratamientos. 


\section{Consentimiento en medicina}

El valor del consentimiento en medicina no es antiguo. Durante mucho tiempo prevaleció la supuesta beneficencia del paternalismo. En el Código de Ética Médica francés de 1947, el artículo 30 manifestaba: «el médico debe esforzarse por imponer la ejecución de su decisión». La evolución de las actitudes sociales y las tecnologías médicas introdujeron el consentimiento informado del paciente como una condición necesaria (pero no suficiente) para garantizar la validez del acto médico y la expresión de la decisión autónoma del paciente. La ley positiva francesa está evolucionando: las leyes del aborto en 1975; la extracción de órganos en 1976; la experimentación médica en 1988 y las leyes bioéticas en 1994, son todas oportunidades para establecer la importancia del consentimiento en la medicina. La ley de Kouchner, del 4 de marzo de 2002, establece que:

No se puede realizar ningún procedimiento o tratamiento médico sin el consentimiento libre e informado de la persona, y este consentimiento se puede retirar en cualquier momento (Artículo L 1111-4 del Código de Salud Pública).

Se hace eco del Código de Ética Médica de 1995, el cual también incorporaba en el artículo 36 la idea de que «se debe buscar el consentimiento de la persona examinada o atendida en todos los casos». Sin embargo, esta obligación presupone la divulgación de información «justa, clara y apropiada» (Artículo R4127-35 del Código de Salud Pública francés) con respecto a la situación clínica del paciente, la utilidad del tratamiento, sus consecuencias, sus beneficios y riesgos, «otras posibles soluciones y las consecuencias previsibles en caso de rechazo» (Artículo L1111-2 del Código de Salud Pública francés), especialmente en cuidados paliativos y ambulatorios. «[El] pronóstico de un estado terminal debe revelarse sólo con precaución, pero los familiares deben ser informados, excepto en circunstancias especiales o si el paciente ha prohibido 
Rechazar y retirar el tratamiento al final de la vida: complejidades éticas...

previamente esta divulgación o designado a los terceros a los que debe hacerse» (Artículo R4127-35 del Código de Salud Pública francés). También debe tenerse en cuenta que en la legislación francesa no hay derecho a disponer del cuerpo de uno (4). Si bien este marco legal es esencial, plantea muchas cuestiones éticas, debido a la profunda vulnerabilidad que experimentan los pacientes al final de la vida y a los problemas psiquiátricos que pueden comprometer la capacidad de toma de decisiones en los pacientes. No obstante, mientras no estemos muertos, estamos vivos, como recuerda Paul Ricoeur en su libro póstumo Vivants jusqu'à la mort 2007 (5). Siempre se mantiene una cierta forma de comunicación en los pacientes, incluso en aquéllos con deficiencias cognitivas, cerca del final de la vida.

El consentimiento es un «yo quiero», escribe Paul Ricoeur en 1950 (6). Es una manifestación esencial de la libertad humana y el resultado de un proceso de toma de decisiones que es racional y emocional, voluntario e involuntario. La etimología nos recuerda que el consentimiento proviene del con-sentire latino, que generalmente se traduce como «estar de acuerdo con». Sin embargo, una traducción más literal y más precisa es «sentir con». Consentir es «comprender intuitivamente, de manera sensible», no sólo lo que está en juego en una situación clínica conmigo mismo (es decir, mis opiniones y mis creencias), sino también con los demás, para aceptar una propuesta de tratamiento médico que no sea perjudicial a mi existencia, con el médico que informa, los familiares y otros cuidadores, porque ellos también son parte de mi existencia. En pacientes con demencia y otras deficiencias cognitivas, nunca debemos olvidar la dimensión emocional de la toma de decisiones y el consentimiento. La forma en que hablamos con los pacientes con demencia, como es el hecho de tomarle una mano, sonreír, colocarse cara a cara, usar la lengua materna del paciente y un lenguaje sencillo, podría contribuir a una cercanía con el paciente a partir de la cual otros, a veces, pueden interpretar la voluntad del paciente y obtener el consentimiento implícito o explícito. 
Sin embargo, el consentimiento sólo es válido siempre y cuando no se revoque. Para una persona con impedimentos cognitivos, las emociones siguen siendo un medio de comunicación que puede cuestionar si esta persona ha dado su consentimiento válido, como en el siguiente caso que involucra a una mujer holandesa de 80 años con demencia. Anteriormente había expresado su deseo de recurrir a la eutanasia cuando consideró que «era el momento adecuado», pero...

A medida que su situación empeoraba, a su esposo le resultaba difícil cuidarla y fue ingresada en un hogar de ancianos.

La documentación médica mostró que a menudo exhibía signos de miedo y enojo, y que deambulaba por el edificio por las noches. El médico principal del hogar de ancianos opinó que estaba sufriendo intolerablemente, pero que ya no estaba en una posición en la que pudiera confirmar que era el momento adecuado para que la eutanasia continuara.

Sin embargo, el médico opinaba que las circunstancias de la mujer dejaban claro que ahora era el momento adecuado.

El médico colocó secretamente un soporífero en su café para calmarla y luego comenzó a administrarle una inyección letal.

Sin embargo, mientras la inyectaban, la mujer se despertó y luchó contra el médico. El papeleo mostró que la única forma en que el médico podía completar la inyección era haciendo que los familiares ayudaran a contener a la paciente.

También se reveló que la paciente dijo varias veces «No quiero morir», en los días previos a su muerte, y que el médico no le había hablado sobre lo que estaba planeado, porque no quería causar una angustia adicional innecesaria. Tampoco le contó lo que había en su café, ya que probablemente causaría más interrupciones en el proceso planeado de eutanasia (7).

Debemos decir que, en este caso, la mujer con demencia revocó sus instrucciones anticipadas y, en sus circunstancias de ese momento, no dio su consentimiento para la eutanasia. La información que se requiere para garantizar el consentimiento informado nunca es neutral, pero en este caso, ni siquiera se dio. 
Rechazar y retirar el tratamiento al final de la vida: complejidades éticas...

La información sobre el final de la vida es crucial al buscar el consentimiento de los pacientes con respecto a su atención médica. Como he escrito en otra parte, la información de salud (diagnóstico, pronóstico...) es «una palabra que se forma, entra dentro del ser donde se forma y deforma, permitiendo que el 'tener' se convierta en un usurpador que cambia la identidad del ser, de tal manera que tener una enfermedad enfermas (8). El psiquiatra JeanJacques Kress señala que «la información no consiste simplemente en transmitir datos, porque lo que el profesional considera como una categoría de conocimiento se convierte en verdad para la existencia única del paciente... Al conocer el pronóstico, la persona se ve afectada en su relación con su destino, con la forma que puede tomar el resto de su vida» (9).

A veces puede ser difícil para los pacientes escuchar la divulgación de una condición que limita la vida. La negación total o parcial no es rara, especialmente cuando ya existe algún grado de deterioro cognitivo experimentado por los pacientes. En todos los casos, este conocimiento produce «efectos subjetivos» que confunden al paciente, a pesar de que tiene la intención de ser «conocimiento sobre el bienestar del paciente», mientras que el médico, a menudo consciente o inconscientemente, espera una «sumisión, como si fuera evidente» (10).

Las ideas de bien, beneficio o beneficencia se relacionan inherentemente con el consentimiento; proponer maltrato no sería ético. Sin embargo, ¿qué debe hacerse si un paciente toma una decisión inaceptable o irresponsable, por ejemplo, cuando el paciente rechaza una atención esencial y razonable que, en caso de que el médico no la brinde, sería médicamente negligente?

Cuando se evalúa que dicho paciente carece de capacidad para tomar decisiones, actuar en el mejor interés de ese paciente es un principio útil, pero esto no debe disimular el hecho de que, a veces, es muy difícil establecer lo que es bueno para otra persona. Es posible que el médico y el paciente no perciban de manera similar lo bueno que se debe hacer, ya que su relación es asimétrica, pues 
este último se encuentra al final de la vida, en una situación de extrema vulnerabilidad, a menudo incapaz de comprender información relevante o capaz de apreciar y juzgar beneficios y cargas. La tensión entre respetar la autonomía y la beneficencia de un paciente hacia este paciente aumenta cuando el paciente está inconsciente y/o carece de capacidad para tomar decisiones y no tiene instrucciones anticipadas. En estas circunstancias, es la acción beneficiosa del profesional de la salud o del cuidador la que debe tener prioridad, escribe Manuel Wolf, aunque esto debe estar «acompañado de garantías sólidas basadas no sólo en la ley, sino también en la educación y el empoderamiento de cada actor en la relación de cuidado, ya sea el profesional de la salud o un simple ciudadano» (3). Ampliar el círculo hermenéutico con respecto a lo que es beneficioso para el paciente para incluir a los familiares y otros cuidadores del paciente es un cambio de paradigma en la toma de decisiones clínicas y éticas. Incorpora un pensamiento complejo. Prohíbe la entrega de decisiones únicamente al médico, pero también requiere la participación de la familia y otros cuidadores.

\section{El papel de los miembros de la familia y otros cui- dadores en el apoyo del consentimiento del paciente}

\section{a) Familia}

El consentimiento del paciente ante el tratamiento protege contra el control por parte de otros del proceso de toma de decisiones en la atención médica, ya sea el médico con su conocimiento o familiares que siempre corren el riesgo de quedar atrapados en la negación del final de la vida de un ser querido o, por el contrario, con la certeza de que todo ya terminó y que ya no es necesario conservar la vida del paciente. Al mismo tiempo, nos guste o no, la presencia de miembros de la familia que están en la vida de una persona afecta el comportamiento y las decisiones del paciente, especial- 
Rechazar y retirar el tratamiento al final de la vida: complejidades éticas...

mente en el caso de personas con discapacidades cognitivas, como la demencia. La familia del paciente a menudo influye en su decisión de continuar o suspender los tratamientos, y el papel de los miembros de la familia se ve afectado, a su vez, por la forma en que ellos mismos experimentan esta situación. Si el paciente regresa a casa para morir, otros parámetros influyen en la experiencia vivida: la idealización frecuente del hogar, pero también la intrusión en los espacios de vida del hogar; el hecho de que a veces el tiempo se ha vuelto demasiado corto, mientras los familiares están exhaustos. Por eso es importante comunicarse bien con las familias, especialmente si los pacientes están inconscientes, carecen de capacidad para tomar decisiones, no han formulado directivas anticipadas o no han designado a una persona de confianza para representarlos o, en Francia, no están cubiertos por un «futuro mandato de protección» (https://www.service-public.fr/particuliers/vosdroits/ F16670).

En Francia, a diferencia de algunos países anglosajones, no hay lugar para el consentimiento sustituto en lugar del paciente (por ejemplo, a través de la tutela). El artículo 8 de la Ley Claeys-Leonetti de 2016 reforzó el peso de las directivas anticipadas, precisamente para no poner el peso de la decisión en la familia. El artículo L. 1111-11 del Código de Salud Pública ahora establece que:

Las directivas anticipadas son vinculantes para el médico ante cualquier decisión de investigar, intervenir o tratar, excepto en casos de emergencia que amenazan la vida y durante el tiempo necesario para una evaluación completa de la situación, y cuando las directivas anticipadas parecen manifiestamente inapropiadas o inconsistentes con la situación médica.

La decisión de negarse a aplicar las instrucciones anticipadas que el médico considera manifiestamente inapropiadas o no de acuerdo con la situación médica del paciente, se toma al final de un procedimiento colegiado definido por el reglamento y se ingresa en el archivo médico. Se dirige a la atención del administrador designado por el paciente o, en su defecto, de la familia o parientes cercanos.

Medicina y Ética - Octubre-Diciembre 2020 - Vol. 31 - Núm. 4 
Por lo tanto, en Francia, la familia no tiene que decidir por el paciente si las indicaciones de las instrucciones anticipadas son inadecuadas. Sólo si el paciente está inconsciente y no tiene instrucciones anticipadas por escrito, el médico preguntará, no sobre la decisión de la familia, sino sobre su testimonio con respecto a los presuntos deseos del paciente. El Artículo 10 de la Ley se refiere al artículo L. 1111-12 del Código de Salud Pública en Francia, y dice lo siguiente:

Cuando una persona, en fase avanzada o terminal de una afección grave e incurable, cualquiera que sea la causa, no puede expresar su voluntad, el médico tiene la obligación de preguntar sobre la expresión de la voluntad expresada por el paciente. En ausencia de instrucciones anticipadas mencionadas en el Artículo L. 1111-11, él tomará el testimonio de la persona de confianza o, en su defecto, cualquier otro testimonio de la familia o parientes.

Por lo tanto, la situación en Francia es bastante diferente del mundo médico anglosajón, donde generalmente se pide a la familia que tome una decisión en sustitución de un paciente que carece de capacidad para tomar decisiones. Sin embargo, cuando los médicos consultan al familiar acerca de los deseos del paciente, debe recordarse la discrepancia entre los deseos del paciente y los de su familia. Además, incluso cuando el paciente tiene directivas anticipadas, no es tan fácil anticipar contingencias futuras al escribir tales directivas anticipadas (11), y la presencia de algún grado de deterioro cognitivo hace que esta tarea sea aún más difícil. Además, cuando se pide a las familias que interpreten los deseos del paciente, a veces se sienten traumatizadas por las experiencias del paciente, y a menudo surgen problemas de comunicación entre los profesionales de la salud y los miembros de la familia (12).

En un estudio se encontró que el 46\% de los encuestados percibieron conflictos durante la estadía de su familiar en la unidad de cuidados intensivos, principalmente debido a problemas de comu- 
Rechazar y retirar el tratamiento al final de la vida: complejidades éticas...

nicación o comportamiento no profesional percibido (como ignorar al cuidador principal en las discusiones sobre el tratamiento). El $48 \%$ de los miembros de la familia informaron sobre la presencia tranquilizadora del clero, y muchos informaron que su médico tratante era la fuente preferida de información y comodidad. JeanJacques Kress señala, en primer lugar, que los profesionales de la salud tienen grandes dificultades tanto para comunicarse sobre el final de la vida, como para ponerse de acuerdo entre ellos y, por lo tanto, después tienen dificultades para ofrecer una representación coherente de sus acciones a las familias (intercambio oral).

Por lo tanto, según Davidson y colaboradores (2017), la atención centrada en la familia (13), (14) -por ejemplo, la empatía mostrada hacia los familiares de un paciente, apoyándoles, comunicándoles cierta información en relación con el paciente, garantizando la libertad de visitas- puede ser una forma decisiva de facilitar la obtención del consentimiento implícito de un paciente con la ayuda de miembros de la familia, o en pacientes evaluados como que no tienen capacidad de toma de decisiones, para fomentar el acuerdo entre los profesionales de la salud y las familias con respecto a una decisión beneficiosa en nombre del paciente.

Nunca debe olvidarse que las familias viven tiempos de angustia. Estando presentes y sin poder salvar a su ser querido, los miembros de la familia están en un momento intermedio difícil. Por un lado están nerviosos mientras esperan la muerte próxima de su ser querido y, por otro, están tensos por el agotamiento, dado que el anuncio de la muerte llega demasiado lento. Esto es particularmente cierto cuando se implementa la sedación continua hasta la muerte del paciente.

La sedación a menudo se presenta como un «ideal hermoso de muerte natural», sin dolor, sin dificultad para respirar, sin agonía visible, sin barreras, sin ansiedad y una muerte sin medicamentos. Sin embargo, esto es una ilusión de una «buena muerte», ya que permanece totalmente bajo control médico. La sedación hace al 
paciente impotente e incapaz de expresar a nadie su experiencia. También hace que la familia se impaciente porque «todo puede terminar pronto», generando en ellos emociones encontradas de culpa y deseo de terminar rápidamente con el sufrimiento del paciente.

\section{b) Cuidadores que no son miembros de la familia}

El trabajo de los cuidadores puede ser esencial para apoyar a los pacientes que carecen de la capacidad de toma de decisiones, con el fin de contribuir a las decisiones de atención médica y dar su consentimiento. Estos cuidadores están cerca del paciente, y a menudo saben lo que está sucediendo ahora con él, mientras que las familias sólo saben lo que sucedió antes.

Cuando un paciente sin instrucciones anticipadas ya no puede expresarse, o porque se encuentra entre momentos de somnolencia y lucidez, a menudo son esos cuidadores quienes primero reconocen los signos de una posible obstinación irrazonable del tratamiento. Su proximidad a la persona, cuyo cuidado implica intimidad corporal, casi siempre les permite sentir empatía y solicitud por el paciente.

Sin embargo, esta proximidad a veces puede ser agotadora. Cuando un paciente sufre; cuando la comunicación entre los médicos, el paciente y los miembros de la familia se vuelve conflictiva; cuando los cuidadores sienten que sus empleadores ya no los apoyan, corren el riesgo de angustia y agotamiento, los cuales son perjudiciales para todos. Éstos sentimientos precipitan decisiones apresuradas y prematuras, o pueden dar lugar a un consentimiento dado por el paciente donde no hay ninguno.

Por lo tanto, es importante promover el bienestar de los cuidadores tanto como sea posible. También significa ser lo más claro posible con respecto a la ineficacia del tratamiento (15). Esto nos recuerda la relevancia de una teología del fracaso. 
Rechazar y retirar el tratamiento al final de la vida: complejidades éticas...

\section{Situaciones clínicas}

Si bien el consentimiento forma el «núcleo» del ejercicio de autonomía de un paciente, puede haber complejidades éticas en torno a la obtención del consentimiento para los tratamientos al final de la vida. Obtener el consentimiento puede ser difícil o imposible en ciertas situaciones. Veamos tres situaciones.

\section{Caso 1. Retirar o limitar el tratamiento de un paciente consciente}

En este caso, ¿cómo informa el profesional de la salud al paciente sobre tal decisión? ¿Podemos detener o limitar el tratamiento sin informar a un paciente que está más o menos consciente y que puede comunicarse? ¿Y sobre qué debe informar el profesional sanitario a este paciente? En teoría, es fácil decirle: «Si continuamos, estamos en una obstinación terapéutica irracional. Entonces debemos detener todo». En la práctica, no es tan simple. ¿Qué significa el mensaje sobre la interrupción o la limitación de los tratamientos para el paciente? ¿Qué pasa si él no quiere ser informado? El artículo L. 1111-2 del Código de Salud Pública francés establece:

Debe respetarse la disposición de una persona a permanecer en la oscuridad sobre un diagnóstico o pronóstico.

Sin embargo, no es fácil determinar si el paciente quiere saber esta información o no; esto requiere una comunicación repetida con el paciente y la posibilidad de que su opinión evolucione durante el progreso de su enfermedad. El tiempo limitado asignado a las actividades de atención, y la híper-especialización de la medicina, junto con la fragmentación de la atención, confunde las consideraciones sobre quién divulga qué información al paciente. Puede haber una gran tentación de asumir que el paciente no quiere ser informado, especialmente en relación con información que es tan difícil de 
vivir existencialmente para el paciente, como la revelación de que él carece de capacidad para tomar decisiones y/o está cercano a la muerte.

¿Deberíamos preguntarnos si el mensaje sobre la interrupción o la limitación del tratamiento es información «útil» que puede ayudar al paciente en su maduración personal a través de esta experiencia al final de la vida? El paciente sólo puede dar su consentimiento a lo que él entiende, pero ¿qué entiende exactamente? ¿Que no hay nada más que hacer? ¿Que va a morir pronto? ¿Que ya pasó su última oportunidad de sobrevivir?

¿No hay una obligación para el médico, con el apoyo de los familiares y otros cuidadores del paciente, de dar prioridad a un mensaje a este paciente que vincule el no abandono y mantener la confianza del paciente, por un lado, y retirar el tratamiento o, incluso, implementar la «sedación continua hasta la muerte»-como lo permite la ley Claeys-Leonetti en Francia-?

En este último caso, también deberá explicarse, que la sedación cortará definitivamente la comunicación del paciente y, una vez sedado, el paciente ya no podrá hacer solicitudes. «¿Cómo le digo al paciente que se está comunicando por última vez? ¿Cómo podemos decirle que seguramente estará presente, pero al mismo tiempo ausente?» (16). Sin mencionar que «la muerte, por predecible que sea, [a menudo] no se desea». ¿Qué sabemos sobre la vida bajo sedación? ¿Qué sabemos acerca de morir y morir bajo sedación? [...] ¿Cómo podemos hablar de «lo indecible y lo impredecible?» (17), (16).

Los síntomas físicos residuales no controlados, sumados al sufrimiento existencial, psicológico o incluso espiritual, realmente pueden conducir a un inmenso cansancio de la vida y contribuir a una forma insoportable de existencia. La demencia que se presenta gradualmente también puede ser aterradora. Los profesionales de la salud y/o los miembros de la familia que rodean al paciente también están encarcelados por un inmenso cansancio. En estas situaciones, ¿el paciente es realmente libre de dar su consentimiento? 
Rechazar y retirar el tratamiento al final de la vida: complejidades éticas...

¿Acaso no le falta alguna opción que sea mejor? No se puede estar éticamente satisfecho con un consentimiento que es un asentimiento vago a las opciones limitadas de la atención médica.

Por otro lado, ¿qué pasa si el paciente se niega a limitar y detener la atención? ¿Puede el médico prescindir de la negativa del paciente por «razones médicas»? Ciertamente es necesario organizar una reunión para discutir tales razones con el paciente, pero el médico debe reconocer la naturaleza progresiva del consentimiento e incorporar esta comprensión más en su relación con el paciente, sin esperar su consentimiento en una reunión. De hecho, buscar el consentimiento es parte de un proceso de comunicación basado en la confianza mutua, la empatía y el apoyo pragmático e interpersonal real, especialmente cuando el paciente tiene impedimentos cognitivos.

Sin embargo, cada vez más, a los profesionales de la salud les resulta difícil conversar con los pacientes sobre este tema y describir claramente las situaciones y decisiones. Service d'Aide Médicale Urgente (Servicio de Asistencia Médica de Emergencia, SAMU) y las unidades de cuidados intensivos en Francia hablan ahora de «pacientes notables», un eufemismo para «no resucitar» (NR). Los médicos pueden considerar la reanimación en caso de enfermedad grave avanzada y progresiva, pero ¿no debería discutirse primero con la persona interesada? Sin embargo, durante un simposio sobre las decisiones médicas al final de la vida, celebrado el 8 de abril de 2019 en el Hospital Europeo Georges-Pompidou de París, se contó la confusa historia de un "paciente tan notable», que ilustra los límites y las preguntas que rodean este término (18). El diario Libération informa sobre los hechos de esta manera:

Es la historia de un hombre que ha sufrido una enfermedad degenerativa por más de 20 años. Recientemente ha tenido otra crisis de insuficiencia respiratoria. Está agotado, cansado, pero no ha tomado ninguna decisión sobre el resto de su vida. Esta vez se sale con la suya y puede irse a casa. Sin embargo, los médicos del hospital, dada la gravedad de la situación clínica de este hombre, deciden celebrar una reunión del 
equipo sobre la continuación de su cuidado. Por extraño que parezca, no se mantiene informada a la familia, pero unos días después reciben una carta que los deja atónitos. Se enteran de que se decidió que, si el paciente regresaba al hospital con urgencia, ya no sería reanimado, y que se iniciaría una sedación larga y continua hasta la muerte. Finalmente, el paciente es reportado como un "paciente notable en SAMU», de modo que se respeta el «procedimiento en caso de nuevas complicaciones respiratorias».

Esto no es legal ni ético. ¡La reunión del equipo para renunciar al tratamiento no puede celebrarse legalmente antes de una futura crisis de salud! El paciente debe dar su consentimiento en el momento de la crisis, si es capaz, o él puede estipular instrucciones anticipadas para cuando carezca de capacidad para tomar decisiones.

Caso 2. Cuando el paciente está inconsciente y no tiene instrucciones anticipadas

El caso de Vincent Lambert en Francia ilustra todos los altibajos de esta situación. El 29 de septiembre de 2008, Vincent Lambert, de 32 años, tuvo un accidente de tránsito que le causó una lesión en la cabeza que lo sumergió en un coma vegetativo, del cual emergió a un estado de conciencia mínima, pero más tarde parecía haber recaído. A pesar de la rehabilitación, no hubo mejoría. El 10 de abril de 2013, su médico concluyó que era víctima de «obstinación terapéutica irracional» en el sentido del artículo L. 1110-5 de Francia, y decidió suspender la alimentación artificial y reducir la hidratación de Vincent Lambert.

Sin embargo, la familia de Lambert estaba dividida: por un lado estaban su esposa Rachel y seis de sus hermanos y hermanas y, por el otro, sus padres Pierre y Viviane y dos de sus hermanos. Rachel argumentó que Vincent, que era enfermero, siempre había rechazado la obstinación mientras practicaba su profesión. Sus padres, católicos muy devotos, argumentaron que su hijo no había llegado al final de su vida y que no debía ser asesinado. Por lo tanto, durante 
Rechazar y retirar el tratamiento al final de la vida: complejidades éticas...

los últimos 11 años ha habido múltiples expertos convocados a juicios, pasando por diferentes tribunales: el Consejo de Estado (2014, 2017, 2019) e incluso el Tribunal Europeo de Derechos Humanos (TEDH) en Estrasburgo (Gran Sala en enero de 2015 y 3 apelaciones para la revisión de esta decisión, todas rechazadas). El procedimiento para suspender la atención fue confirmado por el Tribunal Administrativo de Chalons, confirmado por el Consejo de Estado y el TEDH en 2019. Todas estas decisiones fueron en la misma dirección, a saber: debido a la atrofia cerebral severa y a las muchas lesiones observadas por múltiples investigaciones médicas, «el mantenimiento de la atención y el tratamiento constituye una obstinación irracional, porque sólo tienen el efecto de mantener artificialmente la vida del paciente». (Esta es la definición jurídica oficial de 'obstinación irrazonable' en la ley francesa. Significa que el paciente no está necesariamente al final de su vida). Por otro lado, «el deseo de Vincent Lambert de no ser mantenido con vida, en este caso se establece que se encuentra en un estado en el que ha estado durante diez años». Desesperados, sus padres han apelado al Comité de los Derechos de las Personas con Discapacidad de las Naciones Unidas. ¿Pero es Vincent Lambert una persona discapacitada? Finalmente, el Gobierno francés recordó que, en conformidad con el derecho internacional, no está obligado a esperar la decisión del Comité de Derechos de las Personas con Discapacidad de la ONU.

Vincent fue hospitalizado durante 11 años para mantenerlo con vida. Su esposa era su tutor legal, pero no tuvo nada que decir en esta decisión. Era imposible llegar a un consenso negociado dentro de la familia de Vincent. ¿Qué debería haberse hecho? ¿Debería la ley haber introducido una jerarquía, dando prioridad a la esposa sobre los padres? El 24 de mayo de 2019, Olivier Falorni, miembro del Parlamento de Charente-Maritime, presentó un proyecto de ley en este sentido. El diputado explicó el mismo día en France Bleu (emisora de radio) que «una esposa es con quien hemos vivido voluntariamente; es decir, el cónyuge o pareja, que tiene prioridad en 
términos de tomar decisiones por nosotros sobre cualquier hijo o hijos adultos, padre o padres. Esto se debe a que elegimos a nuestro cónyuge; no necesariamente elegimos a nuestros padres».

Existe la cuestión adicional de quién debería haber pagado por el cuidado continuo de Vincent Lambert. No se trata de evaluar financieramente el valor de la vida de un hombre. La mayoría, incluida la Iglesia Católica, reconoce que la nutrición y la hidratación artificial continua en el contexto de la «obstinación irrazonable» no es, en principio, deseable, porque esta situación impone cargas al paciente que pueden ser desproporcionadas para el beneficio terapéutico de esta persona al final de la vida (ver Instituto Católico Canadiense de Bioética y Asociación Internacional de Bioética Católica) (19). La justicia distributiva, entonces, también se convierte en un problema cuando los recursos sanitarios son limitados. Al buscar un tratamiento que sea moralmente opcional, habría menos recursos disponibles para otras formas de atención médica necesaria; por ejemplo, para la atención primaria de niños y jóvenes.

El caso de Vincent Lambert fue evaluado éticamente de manera diferente por ciertos grupos Pro-Vida y por algunos obispos. Para ellos, se trataba de faltar al respeto a la inviolabilidad de la vida humana. Éstos representaban a pacientes que morían de hambre y sed, como resultado de retirar la nutrición e hidratación artificial en películas y otros medios.

Finalmente, la Cour de Cassation anuló un tribunal de apelaciones que había ordenado a los médicos que mantuvieran vivo al señor Lambert, mientras el Comité de la ONU revisaba su situación. El 2 de julio de 2019 los médicos eliminaron su hidratación y nutrición mientras lo sedaban. Vincent Lambert falleció el 11 de julio de 2019. Para evitar posibles apelaciones, el fiscal de Reims anunció, el mismo día, la apertura de una investigación sobre las causas de muerte y ordenó una autopsia.

Una declaración conjunta de líderes religiosos -rabinos, imanes, pastores, obispos- de Reims (Francia), la ciudad donde murió el señor Vincent Lambert, el 11 de julio de 2019, trajo algo de paz: 
Rechazar y retirar el tratamiento al final de la vida: complejidades éticas...

...Reconocemos sin reservas que es acorde con la dignidad de cada ser humano rechazar todo tratamiento que se considere innecesario, desproporcionado o probable, que cause sufrimiento adicional, siempre que tal decisión no ponga en peligro la vida de ninguna otra persona... Nos gustaría recordar a nuestros conciudadanos que depender de otros para recibir atención o para los actos de la vida cotidiana no significa perder su dignidad... Expresamos nuestra confianza en los médicos de nuestro país. Es necesaria nuestra confianza colectiva en sus capacidades científicas y humanas para que puedan continuar tomando las mejores y más sabias decisiones médicas, al entablar un diálogo genuino con las personas al final de sus vidas o con los familiares de las personas que se han vuelto incapaces de comunicar...

Afortunadamente, no todos los casos son tan éticamente divisorios y controvertidos. En esta situación, aplicar el principio de respetar la autonomía no es suficiente. También se debe considerar el propósito de la atención; es decir, si es por el bien del paciente. Por lo tanto, se debe aplicar el principio de beneficencia. Sin embargo, surge inmediatamente esta pregunta: ¿bajo qué condiciones la beneficencia no es paternalismo? Debe ir acompañado de «garantías sólidas», como señalamos anteriormente, siguiendo a Manuel Wolf.

Sin embargo, al actuar de manera beneficiosa, ¿podrían los profesionales de la salud no involucrar a los familiares del paciente? ¿Qué deberían solicitar de los miembros de la familia? ¿Se puede imponer una decisión judicial en caso de desacuerdo familiar?

\section{Caso 3. Cuando el paciente rechaza a sabiendas el (los) tratamiento(s)}

Para un paciente que es incapaz de tomar decisiones, es muy difícil expresar su rechazo a los tratamientos. Muy a menudo, tanto la familia como los profesionales de la salud acuerdan afirmar que ese paciente no ha entendido lo que está en juego.

Para comprender completamente los problemas, volvamos a leer el testimonio del conocido teólogo alemán (20), que acaba de publicar, con su esposa Irene, un libro titulado Sterben und Lieben (Muriendo y amando) sobre sus experiencias. Irene Mieth tenía dolor 
de espalda intenso y una rápida disminución en el estado general, lo que condujo a un diagnóstico inesperado de cáncer de seno con metástasis. Además de la analgesia en dosis altas, se necesitaba rápidamente una estabilización quirúrgica. Sin embargo, cuando Dietmar buscó más consejos médicos y tratamientos innovadores, su esposa escribió instrucciones anticipadas en las que rechazó cualquier intervención médica (sin nutrición artificial, sin respirador, sin diálisis, sin antibióticos, sin transfusión de sangre...; sólo una analgesia efectiva, aunque acortara la vida y, si es posible, la atención domiciliaria). Dietmar acompañó a Irene durante este periodo oscuro, pero no entendió por qué rechazó los tratamientos recomendados. Escribió que él mismo habría tomado una decisión diferente. Sin embargo, apoyó la decisión de Irene, quien murió seis semanas después del diagnóstico. Le llevó un año entenderlo. Esta situación ilustra una denegación de consentimiento que Irene pudo expresar, que fue atendida y por la cual asumió toda la responsabilidad. Esto permitió que se reconocieran sus deseos libres y que ambos cónyuges entablaran un diálogo único en su profundidad (21).

Sin embargo, rechazar el tratamiento no siempre se experimenta de manera tan positiva, especialmente en el caso de pacientes que se considera que carecen de capacidad para tomar decisiones. Cuando los pacientes rechazan los tratamientos, esto a veces puede dañar profundamente a los miembros de la familia, que podrían considerar tal negativa como enmascarar el suicidio o abandonar a la familia, especialmente cuando el paciente todavía tiene niños pequeños.

Rechazar tratamientos no significa necesariamente rechazar todos los cuidados. El rechazo de un paciente debe conducir a un diálogo en verdad para comprender mejor las implicaciones de dicho rechazo, para tratar de identificar lo que rechaza el paciente y contextualizar el rechazo. ¿Se debe a una falta de comprensión? ¿La negativa carece de claridad? ¿Es una demanda, con presión sobre alguien más para cumplirla? ¿Ha sido influenciado el paciente, 
Rechazar y retirar el tratamiento al final de la vida: complejidades éticas...

y en qué medida, por los miembros de su familia; por afiliaciones religiosas o culturales; por miedos y ansiedades vinculados a ciertas representaciones de tratamiento; por recuerdos «no digeridos» en relación con personas conocidas, o por la animación de la sociedad (22) dirigida a reducir los costos de atención médica?

Un paciente que se considera que carece de capacidad para tomar decisiones podría no ser capaz de expresar argumentos racionales, pero podría romper todos los tubos y dispositivos médicos. El comportamiento repetido de este tipo debería dar lugar a preguntas sobre tales intervenciones, porque este comportamiento podría ser equivalente a las palabras. Un paciente con demencia que se niega de repente a alimentarse, en ausencia de razones médicas, también podría estar expresando su voluntad.

El rechazo de la terapia no debe conducir a una ruptura de la relación de atención médica o alianza terapéutica. Como se recordó en la Opinión $\mathrm{N}^{\circ} 87$ de CCNE ya mencionada (14 de abril de 2005), los médicos no deben estar satisfechos con las decisiones que son demasiado rápidas, inmaduras o desinformadas. Pierre Hum et al. también hablan de «consentimientos encubiertos»: el ideal del consentimiento incondicional y definitivo o, por el contrario, un acuerdo que es demasiado delgado, poco claro, limitado o abstracto. Los autores señalan que el consentimiento del paciente o el rechazo del tratamiento es «siempre parcial, evoluciona y está sujeto a dinámicas relacionales» (23). Este es un punto importante, porque es en las relaciones de confianza donde el consentimiento o rechazo de un paciente puede ser expresado, interpretado por aquellos que lo conocen y, en última instancia, respetado.

Sin embargo, esta alianza terapéutica, negociada después de que un paciente haya tomado una decisión informada de rechazar el tratamiento, sigue siendo frágil, particularmente debido a la híperespecialización médica, y porque los familiares u otros cuidadores podrían no entender la decisión del paciente de rechazar el tratamiento. Los familiares pueden sentirse totalmente confundidos. Podrían sentirse privados de la ayuda y el apoyo que querían para 
que el paciente, su ser querido, sobreviviera. En cambio, el equipo de salud apoya la decisión del paciente de dejar de luchar contra la muerte. ¿Cómo podemos apoyar a estos familiares que no pueden entender la negativa del paciente al tratamiento? Dietmar Mieth habla sobre la fe compartida con su esposa y sobre los recursos espirituales que extrajo de Meister Eckhart. Sin duda, todos deben encontrar personas con quienes hablar, lecturas y alimento espiritual. En el estudio de Abbott mencionado anteriormente, el 48\% de los miembros de la familia informaron sobre la presencia tranquilizadora del clero. Esto no es casualidad.

No debe olvidarse que, a pesar de que Dietmar Mieth apoya la decisión de su esposa, él sólo la entendió un año después de su muerte. En el prólogo de su libro señala:

Ahora, después de más de un año, sé a qué se refería. Porque no puedo morir en sus brazos, como ella murió en los míos. Sin embargo, amar, incluso en la debilidad, es siempre una capa cálida y beneficiosa, que la esperanza y la fe ponen en nuestros hombros.

\section{Conclusión}

Se podrían abordar muchos otros problemas relacionados con el consentimiento sobre el tratamiento al final de la vida. Quizás uno de los más importantes es cómo la representación de la muerte afecta la recepción de información y el consentimiento, de acuerdo con los diferentes actores involucrados en este proceso. ¿Cómo se influye la toma de decisiones al final de la vida, cuando la muerte se representa como aterradora, o mediante la ilusión de una buena muerte tranquila y «natural» a través de la sedación, o por la promesa de una buena muerte, al interrumpir o limitar el tratamiento?

Pero también, ¿qué sucede con el consentimiento en vista de la híper-especialización médica y la fragmentación de la atención entre diferentes estructuras de atención médica y diferentes profesionales de la salud? Por ejemplo, una persona mayor que, en un 
Rechazar y retirar el tratamiento al final de la vida: complejidades éticas...

hogar de ancianos, muestra signos de un derrame cerebral y es ingresada en la sala de emergencias; es revisada por el radiólogo y por una serie de otros profesionales de la salud que podrían no estar interesados en ella como persona, sino en partes de su cuerpo. Debido a que se le considera que carece de capacidad para tomar decisiones, el neurólogo decide transferirla a neurocirugía, pero muere a su llegada. Las diversas investigaciones realizadas sobre ella, buenas en sí mismas, probablemente no se adaptaron a la situación de esta persona mayor, que finalmente murió rodeada de tecnología médica, pero sin los apoyos personales que necesitaba.

Respetar la contribución de un paciente al proceso de toma de decisiones y consentimiento, está en consonancia con el respeto de la autonomía y la dignidad de la persona, especialmente cuando esta persona carece de capacidad para tomar decisiones. Sin embargo, el final de la vida a menudo involucra complejidades éticas que rodean la toma de decisiones y el consentimiento, que es lo que ha querido ilustrar este artículo. Tomar conciencia de esto contribuye a un mayor compromiso con las relaciones sanitarias respetuosas y afectuosas en las que los pacientes participan en la toma de decisiones, y en dar el mayor consentimiento posible durante todo el proceso de atención.

\section{Referencias bibliográficas}

1. CCNE, Comité Nacional Consultivo de Ética francés. Opinión $N^{\circ} 87$. Rechazo al tratamiento y autonomía personal. 14 de abril de 2005. Recuperado en: https:// www.ccne-ethique.fr/en/type_publication/avis?page $=5$

2. Veshi D. Comentarios sobre el caso Lambert: los fallos del Consejo de Estado francés y del Tribunal Europeo de Derechos Humanos. Med Health Care Philos. 20 de junio de 2017; (2): 187-193. Wikipedia Asunto Vincent Lambert. Recuperado en: https://fr.wikipedia.org/wiki/Affaire_Vincent_Lambert (consultado el 9 de junio de 2020). https://doi.org/10.1007/s11019-016-9724-3

3. Wolf M. Consentimiento, ética y dogma. Ética, Medicina y Salud Pública. Enero 1, 2015; (1): 120-4. https://doi.org/10.1016/j.jemep.2014.12.002

Medicina y Ética - Octubre-Diciembre 2020 - Vol. 31 - Núm. 4 
4. Hennette-Vauchez S. ¿Deshacerse de uno mismo? Un análisis del discurso jurídico sobre los derechos de la persona sobre el cuerpo del hijo. París: L'Harmattan; 2004.

5. Ricoeur P. Vivo hasta la muerte. Rastreo de fragmentos. París: Seuil; 2007.

6. Ricoeur P. Filosofía de la voluntad I. Lo voluntario y lo involuntario, París: Aubier, 1950; 1988.

7. Crouch G. Una doctora holandesa drogó el café de una paciente y luego le pidió a su familia que la mantuviera presionada mientras luchaba por no ser asesinada, pero no infringió las leyes de eutanasia del país. Correo Diario. 28 de enero de 2017. Consultado el 10 de julio de 2017. Recuperado en: http://www.dailymail. co.uk/news/article-4166098/Female-Dutch-doctor-drugged-patient-s-coffee.html «\»ixzz4mSevx78w

8. Thiel MJ. Introducción. En Thiel MJ, editor. Entre el dolor y la esperanza. Anunciando enfermedad, discapacidad, muerte. Estrasburgo: Pus, 2006; 5-20.

9. Kress JJ. Ética en psiquiatría: información, consentimiento, decisión. En MarieJo Thiel, editor. ¿Adónde va la medicina? Sentido de las representaciones y prácticas médicas. Estrasburgo: PUS; 2003; 55-67.

10. Kress JJ. Aspectos éticos del consentimiento para la psicoterapia. Ann Psychiatr. 1998; 13 (2): 85-90.

11. Thiel MJ. Capacidad personal para anticipar enfermedades futuras y preferencias de tratamiento. En Lack P, Biller-Andorno N, Brauer S, editores. Directivas avanzadas. Dordecht: Springer. 2014. 17-35. https://doi.org/10.1007/978-94-0077377-6_2

12. Abbott KH, Sago JG, Breen CM, Abernethy AP, Tulsky JA, Familias mirando hacia atrás: un año después de la discusión sobre la retirada o la retención del soporte vital. Crit Care Med. Enero de 2001; 29(1): 197-201. https://doi.org/10.10 97/00003246-200101000-00040

13. Mazaud A. Fin de la vida en la reanimación: vivida primero a cargo de las familias, y luego por los cuidadores. Hasta la muerte acompañando a la vida. Enero, 2019; (136): 95-106. https://doi.org/10.3917/jalmalv.136.0095

14. Davidson JE, Aslakson RA, Long AC, Puntillo KA, Kross EK, Hart J, Cox CE, Wunsch $H$, Wickline MA, Nunnally ME, Netzer G, Kentish-Barnes N, Sprung CL, Hartog CS, Coombs M, Gerritsen RT, Hopkins RO, Franck LS, Skrobik Y, Kon AA, Scruth EA, Harvey MA, Lewis-Newby M, White DB, Swoboda SM, Cooke CR, Levy MM, Azoulay M, Curtis JR. Pautas para la atención centrada en la familia en el neonatal. UCl pediátrica y adulta. Crit Care Med. Enero de 2017; 45(1): 103-128. https://doi.org/10.1097/ccm.0000000000002169

15. Jacquemin D. Bioética, medicina y sufrimiento. Hitos de una teología del fracaso. Québec: Médiaspaul; 2002.

16. Tomczyk M, Viallard ML, Beloucif S, Mamzera MF, Hervé C. Sedación continua hasta la muerte: ¿Cómo informar al paciente y obtener su consentimiento? Medicina Paliativa. 2015; 14;(2): 111-117. https://doi.org/10.1016/j.medpal.2015. 01.003

17. Jankélévitch V. Mort. París: Flammarion; 1977. 
Rechazar y retirar el tratamiento al final de la vida: complejidades éticas...

18. Favereau E. Atención «paciente notable». Liberation, 9 de abril de 2019. Recuperado en: https://www.liberation.fr/france/2019/04/09/attention-patient-remarquable_1720079

19. Instituto Canadiense de Bioética Católica y Asociación Internacional de Bioética Católica. Reflexiones sobre nutrición e hidratación artificial. National Catholic Bioethics Quarterly. Invierno de 2004: 774-782. Recuperado en: https://iacb.ca/ wp-content/uploads/2018/06/ANH.pdf https://doi.org/10.5840/ncbq20044410

20. Mieth D, Mieth I. Sterben und Lieben. Selbstbestimmung bis zuletzt. Friburgo de Brisgovia: Herder Verlag; 2019.

21. Thiel MJ. Tener que morir en nombre de la autonomía. La Croix. 6 de marzo de $2017 ; 26$.

22. Olivier C, Thiel MJ, Fin de la vida a riesgo de expoliación de la autonomía. Esprit. Dic 2017; (440): 124-135. https://doi.org/10.3917/espri.1712.0123

23. Hum P, Boury D, Danel T, Demailly L, Dujardine V, Ethuin C, Lequin F, Pruvot FR, Racine A, Valette P, Vandoolaeghe S, Weil B, Weill S. La negación de la atención: fortalezas y debilidades del consentimiento. Éthique et santé. 2015; (12): 56-63. https://doi.org/10.1016/j.etiqe.2014.09.002 
